\title{
A Corneal Parameter Estimation Method Based on the Minimum Error Method
}

\author{
Zhang Zhengzheng ${ }^{1}$, Zhang Guosheng ${ }^{2}$, Chi Jiannan ${ }^{1}$ \\ ${ }^{1}$ School of Automation and Electrical Engineering, University of Science and Technology, Beijing, PRC \\ ${ }^{2}$ Ministry of Transport of the People's Republic of China, Key Laboratory of Operation Safety Technology on Transport Vehicle Beijing, \\ Beijing, PRC
}

\section{Email address:}

18811348048@163.com (Zhang Zhengzheng), gs.zhang@rioh.cn (Zhang Guosheng), ustbjnc@ustb.edu.cn (Chi Jiannan)

\section{To cite this article:}

Zhang Zhengzheng, Zhang Guosheng, Chi Jiannan. A Corneal Parameter Estimation Method Based on the Minimum Error Method. Science Innovation. Vol. 6, No. 4, 2018, pp. 201-208. doi: 10.11648/j.si.20180604.15

Received: June 24, 2018; Accepted: July 12, 2018; Published: July 20, 2018

\begin{abstract}
In order to solve the problem of high calibration precision in the existing estimation model of corneal parameters, a method of corneal parameter estimation based on minimum error method is proposed in this paper. First, a single camera and dual light source system is built to get the human eye image. And then the center points of the Purkinje spots can be located from the image. The coordinates of the center points is used as the input parameter of the model. Then the radius of the cornea is estimated and substituted into the corneal estimation model. Finally, the center of corneal curvature can be obtained according to the constraint condition. Experimental results show that this method has high precision and strong robustness.
\end{abstract}

Keywords: Curvature Center of Cornea, Estimation of Corneal Parameters, Visual Axis Reconstruction

\section{一种基于最小误差法的角膜参数估计方法}

\author{
张正正 ${ }^{1}$, 张国胜 ${ }^{2}$, 迟健男 ${ }^{1}$ \\ ${ }^{1}$ 自动化学院, 北京科技大学, 北京, 中国 \\ ${ }^{2}$ 北京运输车辆运行安全技术重点实验室, 中华人民共和国交通部, 北京, 中国
}

\section{邮箱}

18811348048@163.com(张正正), gs.zhang@rioh.cn(张国胜), ustbjnc@ustb.edu.cn(迟健男)

\begin{abstract}
摘要: 针对现有的角膜参数估计模型中对系统标定精度要求高的问题, 本文提出了一种基于最小误差法的角膜参数估 计方法。首先本文搭建了单相机双光源系统, 获取人眼图像, 得到普尔钦斑坐标, 并将其作为模型的输入参数, 然后 估计角膜曲率半径, 将其代入角膜估计模型, 根据约束条件求出角膜曲率中心。实验结果表明, 此方法具有精度高、 鲁棒性强的优点。
\end{abstract}

关键词: 角膜曲率中心, 角膜参数估计, 视轴重建

\section{1. 引言}

随着科技的发展，计算机技术和数字相机技术得到了 前所未有的进步。视线追踪是利用机械、电子、光学等各
种检测手段来定位眼睛的特征, 并估计注视点的过程。在 视线追踪系统中, 根据观测者的头部限制可分为基于映射 的2-D视线追踪方法 [3] 和基于模型的 3-D视线追踪方法 [4-6]。2-D视线追踪系统通常指单相机单光源系统, 这种 系统通常由提取睲孔-普尔钦斑矢量和求解注视点映射函 
数两部分组成。首先利用图像处理技术从相机采集的人眼 图像中提取瞳孔中心及普尔钦斑中心的位置, 组成瞳孔普尔钦斑矢量, 再在测试者头部固定的状态下, 由视线追 踪系统在显示器上给出一些标定点, 通过测试者注视这些 标定点来提取瞳孔-普尔钦斑矢量, 利用一组非线性方程 来描述睹孔-普尔钦斑向量与屏幕点之间的映射关系，最 后根据这个映射关系可以实时求出测试者在屏幕上的注 视点。

2-D视线追踪系统只能通过图像的视线特征参数到在 屏幕上落点之间的映射模型求取视线在屏幕上的注视点, 无法获得视线在系统相机坐标系下真实的空间方向。在现 有的算法中, 映射模型都是采用一组非线性多项式来描述, 这种映射模型需要比较复杂的用户标定程序来确定其中 的系数。当使用者的头部位于用户标定位置时, 单相机单 光源系统能够获得较为精确的视线在屏幕上的关注点。但 当使用者头部偏离标定位置时, 单相机单光源系统的视线 关注点估计精度会显著下降。当采用典型的2-D非线性多 项式作为映射模型时, 头部偏离标定位置后对屏幕注视点 精度的影响: 当使用者在上下和左右方向移动头部时, 注 视点的精度变化不大; 当头部远离和靠近摄像机, 即相对 于屏幕前后移动时, 对注视点的精度影响非常大。这是因 为使用者头部上下左右移动时, 图像检测和人眼特征提取 具有较强鲁棒性。而使用者头部远离和靠近相机时, 人眼 参数相对于相机坐标系的 $\mathrm{z}$ 轴来说是变化的, 非线性的二 维平面数学模型无法反应这一参数的变化, 因而对精度影 响很大。

目前，市场上比较成熟的视线追踪设备多数应用了 2-D视线追踪技术，例如瑞典Tobii公司生产的ProX2系列 眼动仪、德国SMI公司生产的RED 500远程眼动仪、美国 ASL公司生产的 501 型眼动仪。

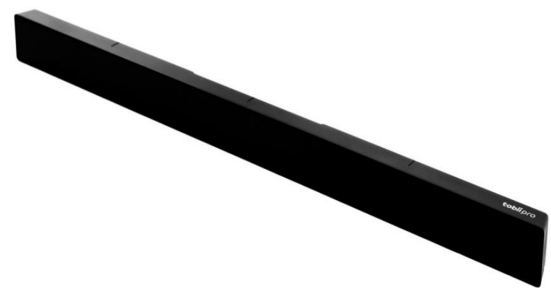

图1 Tobii公司眼动仪。

2-D视线追踪系统技术虽已得到了广泛应用，但其单 目视觉系统无法获得三维信息, 存在一些弊端如: 标定过 程复杂; 追踪的精度不稳定; 在使用过程中要求头部姿势 固定不动等问题。因此, 3-D视线追踪技术应运而生, 其 主要的目的是解决2-D视线追踪技术标定复杂及头部限制 等问题。

三维视线追踪系统能够根据眼球结构和成像模型估 计出在系统相机坐标系下视线的空间方向, 因此这类系统 可以获得自由头动下的视线空间方向, 并且能够简化用户 的标定程序。在单相机双光源系统中, 用户需要标定角膜 曲率中心、睲孔中心、角膜曲率半径这些必要的人眼特征 参数, 这些人眼参数是最终构建三维视线估计模型的基础。 一般来说采用一台高分辨率相机, 配两个光源, 采用三维
几何模型可以实现三维视线方向的检测, 在不大的视场角 范围内可以实现头动状态下的 $1.5^{\circ}$ 的精度。近年来3-D视线 追踪技术发展迅速, 具有干扰小, 易操作, 实用性强的特 点。

本文对视线追踪系统中现有的角膜参数估计模型进 行了研究, 针对此模型对系统标定精度高的问题, 提出了 一种基于最小误差法的角膜参数估计方法, 此方法先是估 计出角膜曲率半径, 然后在增加了角膜曲率半径的约束下 去估计角膜曲率中心, 实验表明此方法具有较强的准确性 和鲁棒性。

\section{3-D视线追踪系统}

3-D视线追踪系统是利用光源及其在角膜上形成的亮 斑图像, 在用户标定阶段确定眼球的不变参数 (如角膜半 径、角膜曲率中心到瞳孔中心的距离等), 在视线估计过 程中估计眼球的变化参数 (如角膜曲率中心坐标等), 然 后根据眼球的结构和成像模型进行视轴重建, 进而获得视 线在相机坐标系下的空间方向, 最后求出视线在屏幕上的 注视点[4-6]。

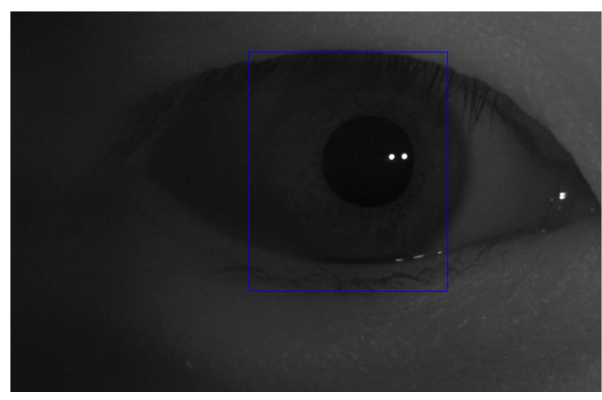

图2 人眼图像。

三维视线估计是根据人眼的特征参数和视线模型来 估计出人眼视线的空间方向, 进而通过视线与注视屏幕的 交点来估计出人眼注视点的空间位置。三维视线估计具有 以下几个优点:

1) 采用立体视觉, 可以检测使用者头部的空间位置, 允许使用者头部自然运动;

2) 采用几何模型进行视线估计, 映射函数不依赖于 标定位置, 视线估计的精度不会随着头部远离标定位置而 下降;

3) 只需要标定视线与眼球光轴的夹角, 不依靠标定 来确定眼睛特征参数与视线的映射函数, 标定过程简单, 标定点数少, 在双相机双光源系统的条件下可以做到单点 标定;

4）视线估计不依赖于屏幕位置、大小和形状，可以 计算视线在任何物体上的注视点, 并且不需要重新标定视 线估计函数。

3-D视线追踪系统一般来说指的是单相机多光源系统 和多相机多光源视线追踪系统, 并且至少需要一个相机配 两个或者两个以上的光源才能够精准测出三维的空间方 向。本文基于单相机双光源的3-D视线追踪系统如下图3 
所示, 主要可分为三个部分: 图像采集、人眼参数获取和 注视点估计。

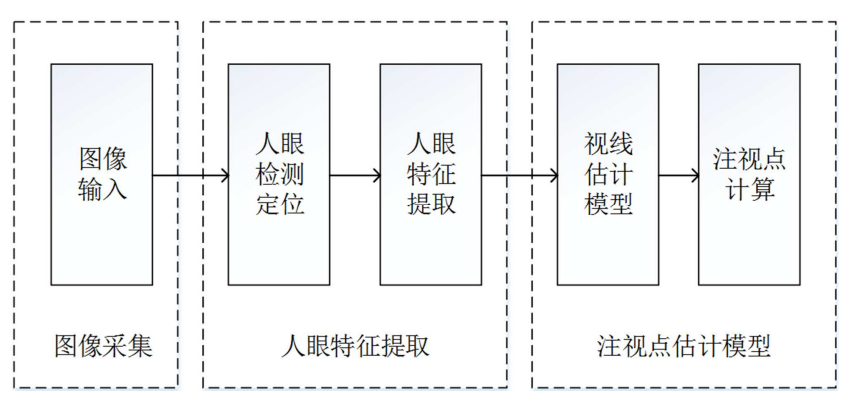

图3 3-D视线追踪系统流程图。

单相机视线追踪系统由一个相机采集人脸图像, 提取 眼睛的视线参数, 其中包括普尔钦斑中心、瞳孔中心、瞳 孔外边界、虹膜中心和虹膜外边界。将提取到的人眼特征 参数作为三维视线立体模型的输入, 采用本文改进的角膜 估计模型可以估计出人眼的角膜曲率中心和半径, 以及估

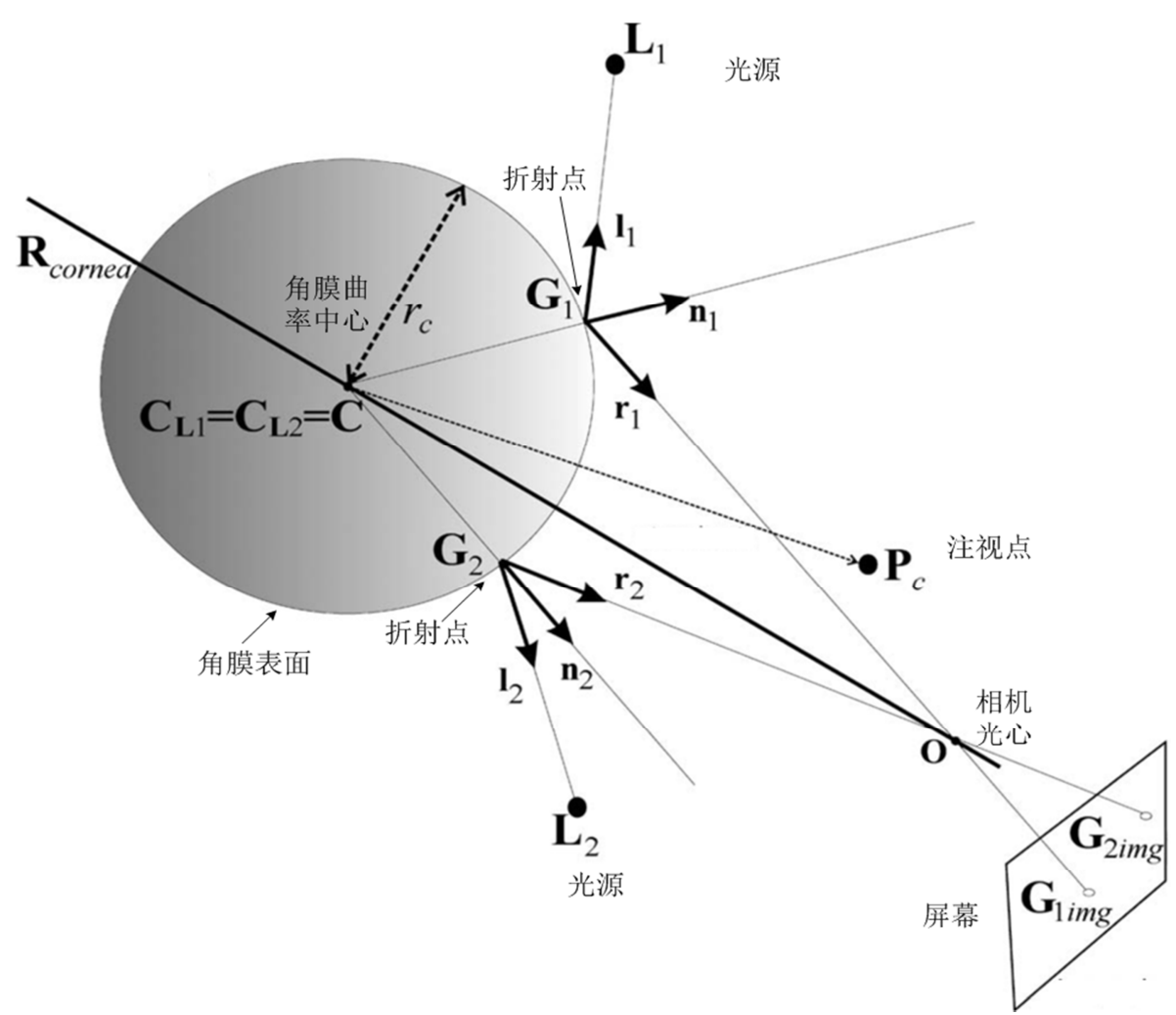

图4 角膜表面反射原理图。
计出瞳孔中心的空间位置, 从而获得人眼光轴的空间方向, 再利用用户标定方法标定出的Kappa角, 可获得人眼视轴 的空间方向。视轴与屏幕的交点, 即为人眼视线在屏幕上 的注视点。

\section{3. 角膜参数估计模型}

\section{1. 角膜参数估计的数学模型}

人眼角膜参数的估计是 $3-\mathrm{D}$ 视线追踪系统中三维视线 方向估计的关键, 角膜参数估计算法的优劣直接关系到 3-D视线追踪系统中光轴方向的精度, 进而影响注视点的 精度, 其中角膜曲率中心坐标及角膜曲率半径的获取是非 常重要的一步。

在单相机双光源系统中角膜参数数学模型是根据眼 球的成像模型, 光的反射原理和点在三维空间中的几何关 系而建立的[14], 其原理如图4所示。
在图 4 中, $\mathrm{C}$ 为角膜曲率中心, $r_{c}$ 为角膜外表面半径, $\mathrm{L}_{1}, \mathrm{~L}_{2}$ 为两个近红外光源, $\mathrm{O}$ 为相机的光心, $G_{1}, G_{2}$ 为光 源照射眼球在角膜表面的反射点, $\mathrm{G}_{1 \mathrm{img}}, \mathrm{G}_{2 \mathrm{img}}$ 为反射点 $\mathrm{G}_{1}, \mathrm{G}_{2}$ 在相机中成像。由光源 $\mathrm{L}_{1}$ 照向眼球的光线经角膜外 表面上的 $G_{1}$ 点反射后在相机中成像 $G_{1 i m g}$, 因此点 $\mathrm{G}_{1}, \mathrm{O}, \mathrm{G}_{1 \mathrm{img}}$ 三点共线, 用向量表示为:

$$
\mathrm{G}_{1}=\mathrm{O}+\mathrm{k}_{1}\left(\mathrm{O}-\mathrm{G}_{1 \mathrm{img}}\right)
$$

同理, 对于光源 $\mathrm{L}_{2}$ 可得：

$$
\mathrm{G}_{2}=\mathrm{O}+\mathrm{k}_{2}\left(\mathrm{O}-\mathrm{G}_{2 \mathrm{img}}\right)
$$

其中, $k_{1}$ 和 $k_{2}$ 为常数。

由于角膜外表面上的反射点 $\mathrm{G}_{1}, \mathrm{G}_{2}$ 到角膜曲率中心 $\mathrm{C}$ 点的距离为角膜曲率半径 $r_{c}$, 故有: 


$$
\begin{aligned}
& \left\|\mathrm{G}_{1}-\mathrm{C}\right\|=\mathrm{r}_{\mathrm{c}} \\
& \left\|\mathrm{G}_{2}-\mathrm{C}\right\|=\mathrm{r}_{\mathrm{c}}
\end{aligned}
$$

一般设定人眼的角膜曲率半径 $r_{c}$ 为常数。

因此根据光的反射原理, 光的反射要满足两个条件: 1)入射光线, 反射光线和法线三线共面; 2)入射角等于反 射角。对于光源 $\mathrm{L}_{1}$ 得:

$$
\mathrm{r}_{1}+\mathrm{l}_{1}=2\left(\mathrm{n}_{1} \cdot \mathrm{l}_{1}\right) \cdot \mathrm{n}_{1}
$$

其中, $r_{1}, l_{1}$ 和 $n_{1}$ 为单位向量, 可得:

$$
\begin{aligned}
& \mathrm{r}_{1}=\frac{\left(\mathrm{G}_{1}-\mathrm{O}\right)}{\left\|\mathrm{G}_{1}-\mathrm{O}\right\|} \\
& \mathrm{l}_{1}=\frac{\left(\mathrm{G}_{1}-\mathrm{L}_{1}\right)}{\left\|\mathrm{G}_{1}-\mathrm{L}_{1}\right\|} \\
& \mathrm{n}_{1}=\frac{\left(\mathrm{G}_{1}-\mathrm{C}\right)}{\left\|\mathrm{G}_{1}-\mathrm{C}\right\|}
\end{aligned}
$$

对于光源 $\mathrm{L}_{2}$, 根据光的反射原理得:

$$
\mathrm{r}_{2}+\mathrm{l}_{2}=2\left(\mathrm{n}_{2} \cdot \mathrm{l}_{2}\right) \cdot \mathrm{n}_{2}
$$

其中, $r_{2}, l_{2}$ 和 $n_{2}$ 为单位向量, 可得:

$$
\begin{aligned}
& \mathrm{r}_{2}=\frac{\left(\mathrm{G}_{2}-\mathrm{O}\right)}{\left\|\mathrm{G}_{2}-\mathrm{O}\right\|} \\
& \mathrm{l}_{2}=\frac{\left(\mathrm{G}_{2}-\mathrm{L}_{2}\right)}{\left\|\mathrm{G}_{2}-\mathrm{L}_{2}\right\|} \\
& \mathrm{n}_{2}=\frac{\left(\mathrm{G}_{2}-\mathrm{C}\right)}{\left\|\mathrm{G}_{2}-\mathrm{C}\right\|}
\end{aligned}
$$

将式 (5) 和式 (7) 分别带入式 (4) 和式 (6) 中得:

$$
\begin{aligned}
& \frac{\mathbf{O}-\mathbf{G}_{1}}{\left\|\mathbf{O}-\mathbf{G}_{1}\right\|}+\frac{\mathbf{L}_{1}-\mathbf{G}_{1}}{\left\|\mathbf{L}_{1}-\mathbf{G}_{1}\right\|}=\mathbf{2} *\left(\frac{\mathbf{G}_{1}-\mathbf{C}}{\left\|\mathbf{G}_{1}-\mathbf{C}\right\|} \bullet \frac{\mathbf{L}_{1}-\mathbf{G}_{1}}{\left\|\mathbf{L}_{1}-\mathbf{G}_{1}\right\|}\right) \times \frac{\mathbf{G}_{1}-\mathbf{C}}{\left\|\mathbf{G}_{1}-\mathbf{C}\right\|} \\
& \frac{\mathbf{O}-\mathbf{G}_{2}}{\left\|\mathbf{O}-\mathbf{G}_{2}\right\|}+\frac{\mathbf{L}_{2}-\mathbf{G}_{2}}{\left\|\mathbf{L}_{2}-\mathbf{G}_{2}\right\|}=\mathbf{2} *\left(\frac{\mathbf{G}_{2}-\mathbf{C}}{\left\|\mathbf{G}_{2}-\mathbf{C}\right\|} \cdot \frac{\mathbf{L}_{2}-\mathbf{G}_{2}}{\left\|\mathbf{L}_{2}-\mathbf{G}_{2}\right\|}\right) \times \frac{\mathbf{G}_{2}-\mathbf{C}}{\left\|\mathbf{G}_{2}-\mathbf{C}\right\|}
\end{aligned}
$$

由光的反射条件1）可知 $\mathrm{L}_{1}, \mathrm{G}_{1}, \mathrm{C}$ 和 $\mathrm{G}_{1 \mathrm{img}}$ 四点共面, $\mathrm{L}_{2}, \mathrm{G}_{2}, \mathrm{C}$ 和 $\mathrm{G}_{2 \mathrm{img}}$ 四点共面[12], 可表示为:

$$
\begin{aligned}
& \left(\mathrm{L}_{1}-\mathrm{O}\right) \times\left(\mathrm{G}_{1}-\mathrm{O}\right) \cdot(\mathrm{C}-\mathrm{O})=0 \\
& \left(\mathrm{~L}_{2}-\mathrm{O}\right) \times\left(\mathrm{G}_{2}-\mathrm{O}\right) \cdot(\mathrm{C}-\mathrm{O})=0
\end{aligned}
$$

将式（1）和式（2）带入式（9）, 可得:

$$
\begin{aligned}
& \left(\mathrm{L}_{1}-\mathrm{O}\right) \times\left(\mathrm{G}_{1 \mathrm{img}}-\mathrm{O}\right) \cdot(\mathrm{C}-\mathrm{O})=0 \\
& \left(\mathrm{~L}_{2}-\mathrm{O}\right) \times\left(\mathrm{G}_{2 \mathrm{img}}-\mathrm{O}\right) \cdot(\mathrm{C}-\mathrm{O})=0
\end{aligned}
$$

由此可知, $\mathrm{L}_{1}, \mathrm{G}_{1}, \mathrm{G}_{1 \mathrm{img}}$ 与 $\mathrm{L}_{2}, \mathrm{G}_{2}, \mathrm{G}_{2 \mathrm{img}}$ 分别共面且 $\mathrm{CO}$ 为两平面的交线, 故式 (10) 可以表示为:

$$
\left[\begin{array}{c}
\left(\mathrm{L}_{1}-\mathrm{O}\right) \times\left(\mathrm{G}_{1 \mathrm{img}}-\mathrm{O}\right)^{\mathrm{T}} \\
\left(\mathrm{L}_{2}-\mathrm{O}\right) \times\left(\mathrm{G}_{2 \mathrm{img}}-\mathrm{O}\right)^{\mathrm{T}}
\end{array}\right](\mathrm{C}-\mathrm{O})=0
$$

式 (11) 中矩阵记为 $\mathrm{M}$, 化简为:

$$
\mathrm{C}-\mathrm{O}=\mathrm{k}_{3} \mathrm{e}_{\text {norm }}
$$

其中, $e_{n o r m}$ 为单位向量, 由向量 $\left(\mathrm{L}_{1}-\mathrm{O}\right) \times\left(\mathrm{G}_{1 \mathrm{img}}-\mathrm{O}\right)$ 和 向量 $\left(\mathrm{L}_{2}-\mathrm{O}\right) \times\left(\mathrm{G}_{2 \mathrm{img}}-\mathrm{O}\right)$ 可以得到:

$$
\mathrm{e}_{\text {norm }}=\frac{\mathrm{e}}{\|\mathrm{e}\|}
$$

其中, $\mathrm{e}=\left[\left(\mathrm{L}_{2}-\mathrm{O}\right) \times\left(\mathrm{G}_{1 \text { img }}-\mathrm{O}\right)\right] \times\left[\left(\mathrm{L}_{2}-\mathrm{O}\right) \times\left(\mathrm{G}_{2 \text { img }}-\mathrm{O}\right)\right]$ 。 通过式 (13), 则点 $\mathrm{C}$ 的坐标可以表示为:

$$
\mathrm{C}=\mathrm{O}+\mathrm{k}_{3} \mathrm{e}_{\text {norm }}
$$

则式 (3) 和式 (8) 可组成为包含三个未知量 $\mathrm{k}_{1}, \mathrm{k}_{2}, \mathrm{k}_{3}$ 的非线性方程组。但是由于非线性方程组模型求解比较困 难, 可以将其转换为优化问题。所得方程组如下:

$$
\begin{aligned}
& f_{1}=2 *\left(\frac{G_{1}-C}{\left\|G_{1}-C\right\|} \cdot \frac{L_{1}-G_{1}}{\left\|L_{1}-G_{1}\right\|}\right) \times \frac{G_{1}-C}{\left\|G_{1}-C\right\|}-\left(\frac{o-G_{1}}{\left\|\mathbf{O}-G_{1}\right\|}+\frac{L_{1}-G_{1}}{\left\|L_{1}-G_{1}\right\|}\right) \\
& f_{2}=2 *\left(\frac{G_{2}-C}{\left\|G_{2}-C\right\|} \cdot \frac{L_{2}-G_{2}}{\left\|L_{2}-G_{2}\right\|}\right) \times \frac{G_{2}-C}{\left\|G_{2}-C\right\|}-\left(\frac{o-G_{2}}{\left\|\mathbf{O}-G_{2}\right\|}+\frac{L_{2}-G_{2}}{\left\|L_{2}-G_{2}\right\|}\right) \\
& f_{3}=\left\|G_{1}-C\right\|-\left\|G_{2}-C\right\|
\end{aligned}
$$

转为无约束优化问题（简称 CCCUO ), 得:

$$
\left\{\begin{array}{c}
\min F(x, y, z)=\sum_{i=1} f_{i}^{2} \\
\text { s.t. } \quad(x, y, z) \in R^{3} \\
\text { s.t. } \quad r_{c}=R
\end{array}\right.
$$

利用最优化原理, 当 $\mathrm{F}\left(\mathrm{k}_{1}, \mathrm{k}_{2}, \mathrm{k}_{3}\right)$ 的最小值为 0 时, 对 应 $\mathrm{k}_{1}, \mathrm{k}_{2}, \mathrm{k}_{3}$ 即为模型的解, 将 $\mathrm{k}_{1}, \mathrm{k}_{3}$ 带入式 (1) 和式 (14) 即可求得角膜曲率中心 $\mathrm{C}$ 的坐标和角膜曲率半径 $\mathrm{r}_{\mathrm{c}}$ 。

\section{2. 基于最小误差法的角膜估计模型}

在实际测量中, 这种求解方法对光源 $\mathrm{L}_{1}$ 和 $\mathrm{L}_{2}$ 的位置 精度要求较高, 由3-D视线追踪系统的系统标定过程中可 知这两个光源相对于系统相机的空间位置很难精确标定, 导致其在角膜外表面出发生反射时, 两条反射法线为不相 交的异面直线, 导致估计出的角膜曲率中心和半径存在误 差。并且此非线性无约束方程组存在病态问题, 即当系统 中光源位置的标定存在较小误差时对估计出的角膜特征 
参数反而产生较大的偏差, 因此这种方法很难构建具有高 检测精度的视线追踪系统。

本文研究发现, 当在上述的角膜参数估计模型中增加 角膜外表面半径 $r_{c}$ 的约束后, 可以获得较高精度的角膜曲 率中心 $\mathrm{C}$ 的空间位置。所以设法去标定出角膜外表面半径 $r_{c}$ 的数值是获得更高精度角膜曲率中心 $\mathrm{C}$ 的空间位置的 更好途径。

通过研究球面镜成像原理, 本文提出了一种基于最小 误差法的角膜参数求解方法。其原理如下:

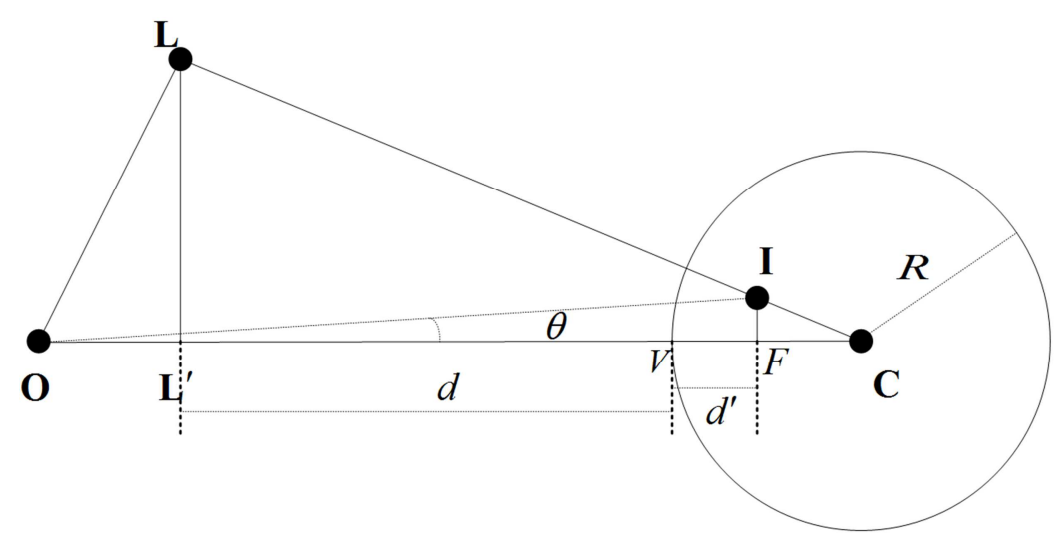

图5 球面镜成像原理。

如图5所示, 根据球面镜成像原理, 对于近轴光源 $\mathrm{L}$ 来 表面, 所以这一球面的焦距就为其曲率半径的一半。由于 说, 其会在角膜外表面形成虚像 I。依据球面镜成像原理, 有以下等式关系:

$$
\frac{1}{d}+\frac{1}{d^{\prime}}=\frac{1}{f}=\frac{2}{R}
$$

其中, $\mathrm{d}$ 可以视为近光轴光源 $\mathrm{L}$ 距球面镜表面的物距, 而 $\mathrm{d}^{\prime}$ 可以视为像距。由于角膜外表面可以近似为球面镜的

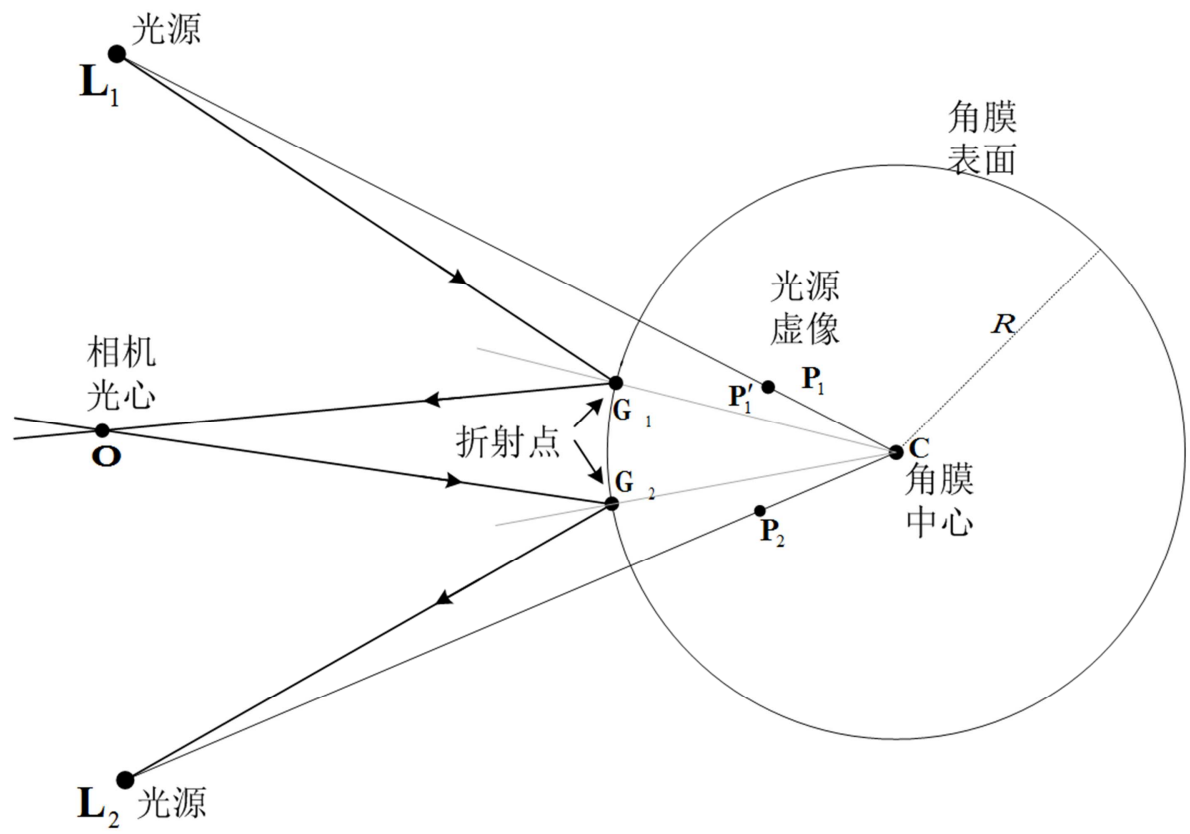

图6 眼球角膜虚像成像原理图。

在图4中, 光源 $\mathrm{L}_{1}$ 在角膜内成的虚像为 $\mathrm{P}_{1}^{\prime}$, 其约位于 半径 $\frac{\mathrm{R}}{2}$ 处, 在直线 $\mathrm{CL}_{1}$ 上距角膜曲率中心 $\mathrm{C}$ 点距离为 $\frac{\mathrm{R}}{2}$ 的点为 $\mathrm{P}_{1}$, 则由式可得, $\left\|\mathrm{P}_{1} \mathrm{P}_{1}^{\prime}\right\|$ 约等于零。当假设的角膜 $d \gg d^{\prime}$, 可以认为光源成像于图示 $\frac{R}{2}$ 处。

在单相机双光源3-D视线追踪系统中, 其光源成虚像 如图6所示: 


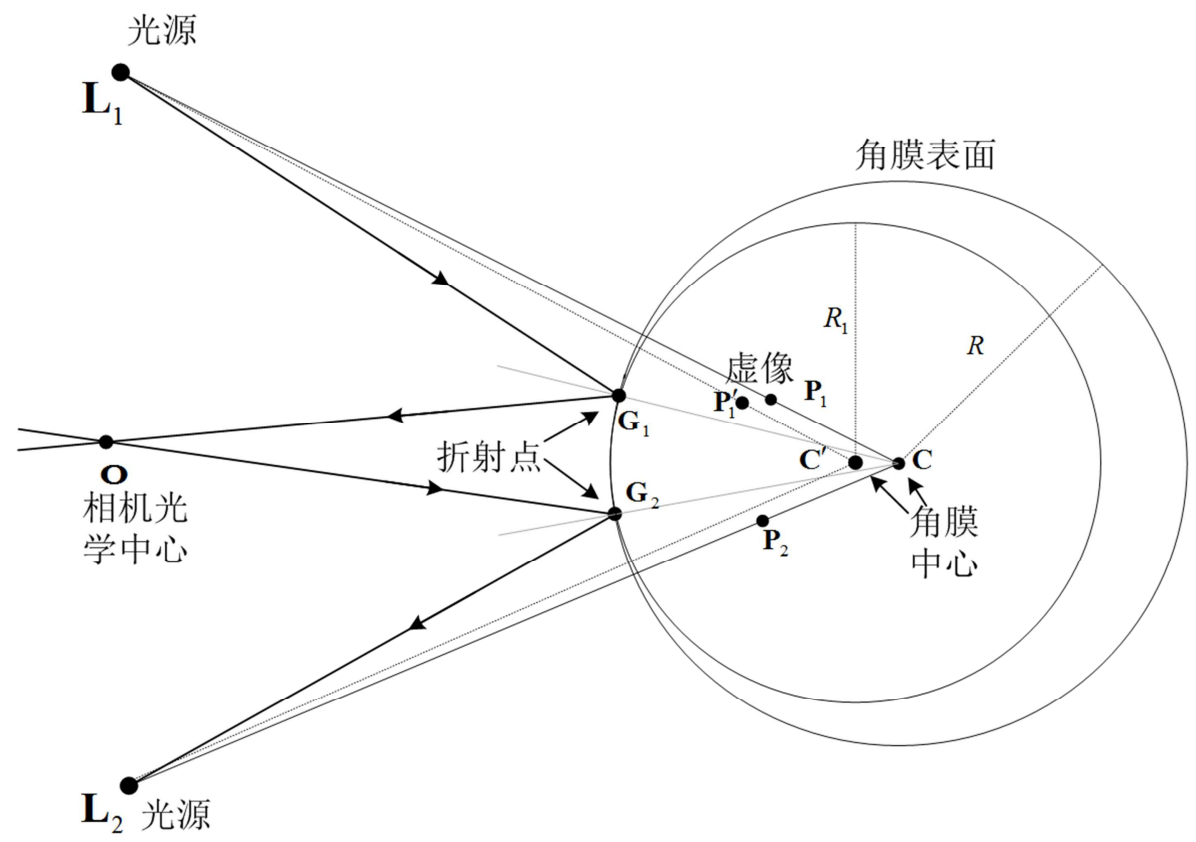

图7 角膜半径不同时的误差原理图。

在视线追踪系统中, 光源空间位置标定的误差, 以及 图像处理方面的误差, 导致两个光源的反射法线不相交, 并且角膜外表面的曲率中心 $\mathrm{C}$ 点不在两个平面相交的直 线上, 因此采用原有模型无法精确地求解出角膜曲率中心 和半径。

所以本文在这里对上述模型进行了改进, 在上述无约 束优化中添加角膜外表面半径 $r$, 将无约束优化改进为有 约束优化, 形式如下:

$$
\mathrm{f}_{3}=\left\|\mathrm{G}_{1}-\mathrm{C}\right\|+\left\|\mathrm{G}_{2}-\mathrm{C}\right\|-2 * \mathrm{r}_{\mathrm{c}}
$$

将模型转为有约束优化问题:

$$
\left\{\begin{aligned}
\min & F(x, y, z)=\sum_{i=1} f_{i}^{2} \\
\text { s.t. } & (x, y, z) \in R^{3} \\
\text { s.t. } & r_{c}=R
\end{aligned}\right.
$$

其中, $(\mathrm{x}, \mathrm{y}, \mathrm{z})$ 参数为角膜曲率中心的 $\mathrm{C}$ 的坐标, $\mathrm{R}$ 为 角膜外表面的半径, 并且 $\mathrm{R}$ 已知。因此利用最优化求解算 法, 当 $\mathrm{F}(\mathrm{x}, \mathrm{y}, \mathrm{z})$ 的最小值为 0 时, 所对应的 $(\mathrm{x}, \mathrm{y}, \mathrm{z})$ 即为模型 的解。

算法分为两个阶段, 第一阶段为求取角膜外表面半径, 用网格搜索法 [9]进行全局搜索, 每一个值都是问题的候选 解, 由改进模型的目标函数构建误差函数, 寻求使误差函 数最小的值, 即为角膜曲率半径。第二阶段是将网格搜索 法得到的角膜外表面半径作为上述改进的有约束模型的 约束, 用LM (Levenberg Marquardt method) 算法 [8]求解, 将此解作为问题的最终解, 最后得到角膜曲率中心 $\mathrm{C}$ 的坐 标。

在第一阶段, 采用如下误差函数:

$$
\left\{\begin{array}{c}
\operatorname{err}(\mathrm{r}, \mathrm{x}, \mathrm{y}, \mathrm{z})=\left\|\mathrm{P}_{1} \mathrm{P}_{1}^{\prime}\right\|+\left\|\mathrm{P}_{2} \mathrm{P}_{2}^{\prime}\right\| \\
\hat{\mathrm{r}}=\underset{\mathrm{r}}{\operatorname{argmin}} \operatorname{err}(\mathrm{r}, \mathrm{x}, \mathrm{y}, \mathrm{z})
\end{array}\right.
$$

其算法步骤如下:

1）标定出光源位置及普尔钦斑位置;

2) 采用网格搜索法, 首先给角膜外表面半径赋初始 值 $r=7.00 \mathrm{~mm}$, 采用有约束的角膜曲率中心求解算法, 计算 出角膜曲率中心 $\mathrm{C}$;

3) 根据球与直线的交点, 求解出角膜上的反射点 $\mathrm{G}_{1}, \mathrm{G}_{2}$;

4）根据 $\mathrm{G}_{1}, \mathrm{G}_{2}, \mathrm{C}$ 三点坐标, 计算出半径中心点 $\mathrm{P}_{1}, \mathrm{P}_{2}$ 的空间坐标;

5) 根据公式（17）计算出光源虚像 $\mathrm{P}_{1}^{\prime}, \mathrm{P}_{2}^{\prime}$ 点的空间位 置;

6) 计算误差: err $=\left\|\mathrm{P}_{1} \mathrm{P}_{1}^{\prime}\right\|+\left\|\mathrm{P}_{2} \mathrm{P}_{2}^{\prime}\right\|$;

7）重复第二步, 赋值 $r=7.00 \mathrm{~mm}+0.02 \mathrm{~mm}$, 直到找到 误差最小的半径 $r$, 即为角膜曲率半径, 最终求出角膜中 心 $\mathrm{C}$ 。

\section{4. 实验与分析}

针对本文提出的一种基于最小误差法的角膜参数估 计算法, 通过实验来分析验证本文改进的算法的准确性及 鲁棒性。

对眼球、相机和光源进行三维场景建模, 以相机光学 中心为系统坐标系原点 $O(0,0,0), X-Y$ 轴平行于相机成像 平面, 垂直与相机成像平面指向眼球的方向为 $\mathrm{Z}$ 轴正方向。 参数设定: 相机的焦距 $\mathrm{f}$ 为 $10 \mathrm{~mm}$, 角膜曲率中心位置 $\mathrm{C}$ 点 为 $(0.000,0.000,600.000)$, 角膜曲率半径 $r_{c}$ 为 $8 \mathrm{~mm}$, 眼球半 
径 $R$ 为 $12 \mathrm{~mm}$, 光源 $\mathrm{L}_{1}$ 的坐标为(74.254,44.096,30.525), 光源 $\mathrm{L}_{2}$ 的坐标为 $(-128.993,-23.565,32.826)$; 光源 $\mathrm{L}_{1}$ 在相 机中的成像 $\mathrm{G}_{1 \mathrm{img}}$ 点坐标为 $(-0.008,-0.005,-10.000)$, 光源
$\mathrm{L}_{2}$ 在相机中的成像 $\mathrm{G}_{2 \mathrm{img}}$ 点坐标为 $(0.015,0.003,-10.000)$ (单位: $\mathrm{mm}$ )。

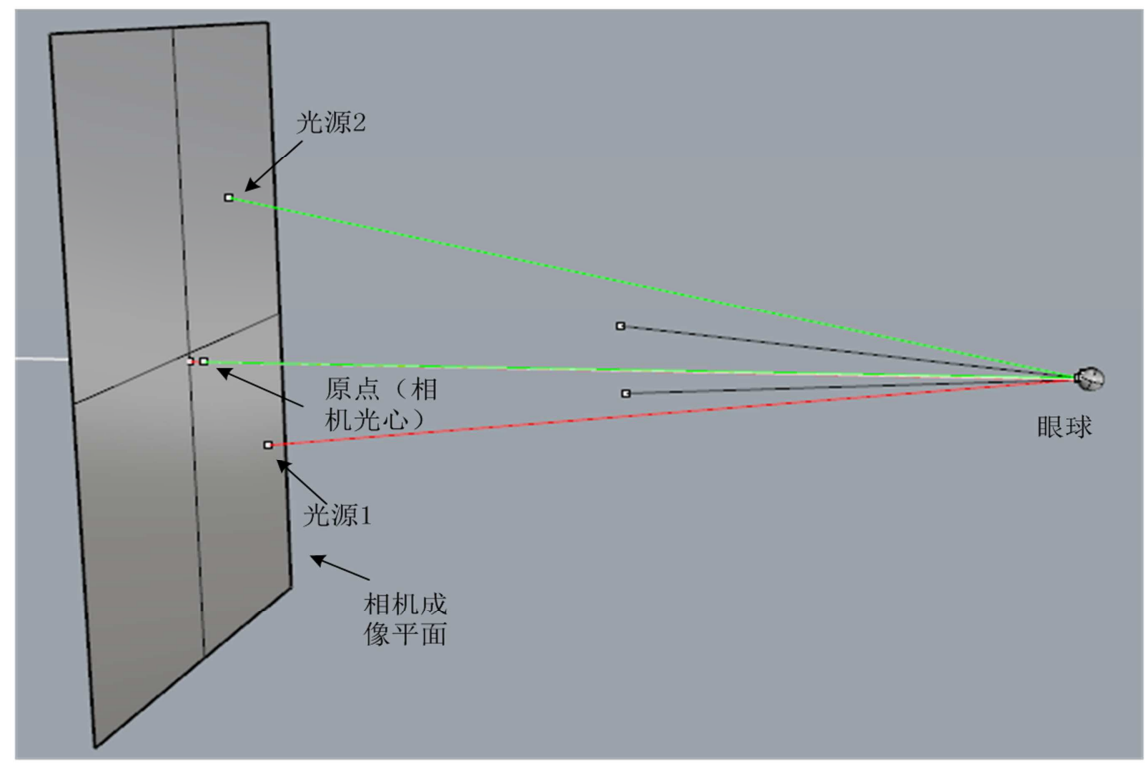

图8 角膜参数实验模型。

\section{1. 准确性}

为验证本文提出的改进的角膜参数估计算法的准确性, 使用仿真模型中的数据, 所得实验结果与原模型的实验结 果对比, 如表1所示:

表1 光源位置精确测量标定(单位: $\mathrm{mm}$ )。

\begin{tabular}{llll}
\hline 原模型 & & 本文改进的模型 & \\
\hline 角膜曲率中心点 $C$ 的空间位置 & 角膜曲率半径 & 角膜曲率中心点 $C$ 的空间位置 & 角膜曲率半径 \\
\hline$(-0.2903,0.0503,605.3548)$ & 7.958 & $(-0.0065,-0.0031,600.2671)$ & 8.000 \\
\hline
\end{tabular}

\section{2. 鲁棒性}

在实际系统中, 系统标定出的光源 $\mathrm{L}_{1}, \mathrm{~L}_{2}$ 的空间位置会有误差, 由于病态问题的存在, 在求解非线性约束最优解 的过程中其对角膜参数的估计影响很大。高精度的视线追踪系统必须具有强鲁棒性。

为验证本文改进的模型对实际系统的鲁棒性, 这里对仿真模型中的相关参数进行了合理的假设, 即对光源 $\mathrm{L}_{1}$ 和 $\mathrm{L}_{2}$ 的空间位置上添加了方差为 $2 \mathrm{~mm}$ 的噪声, 并将实验结果与采用原模型的实验结果进行了对比, 其结果如表 2 所示:

表2 光源位置精确测量标定(单位: $\mathrm{mm}$ )。

\begin{tabular}{llll}
\hline 原模型 & & 本文改进的模型 & 角膜半径 \\
\hline 角膜中心点 $C$ 的空间位置 & 角膜半径 & 角膜中心点 $C$ 的空间位置 & 8.000 \\
\hline$(-51.5252,0.2287,202.4223)$ & 3.191 & $(-0.0106,-0.0095,599.6891)$ & 8.000 \\
$(-6.6005,5.1189,-700.9962)$ & 11.711 & $(-0.0075,-0.0071,598.0762)$ & 8.000 \\
$(-20.5018,-0.1454,358.5796)$ & 12.023 & $(-0.0002,-0.0019,599.7494)$ & 8.000 \\
$(-9.8224,-3.9852,396.1268)$ & 38.869 & $(-0.0070,-0.0146,600.0532)$ & 8.000 \\
$(-10.5574,0.0087,502.4185)$ & 7.545 & $(0.0014,-0.0010,600.2397)$ & 8.000 \\
$(-283.4954,0.3752,162.8065)$ & 2.364 & $(0.0127,-0.0046,600.8709)$ & 8.000 \\
$(-23.8914,0.3167,334.3193)$ & 15.017 & $(0.0042,-0.0005,600.3257)$ & 8.000 \\
$(-33.0567,0.2406,391.7463)$ & 2.861 & $(-0.0104,-0.0073,599.0777)$ & 8.000 \\
$(-27.5214,4.2683,450.4229)$ & 12.636 & $(0.0115,0.0021,599.5289)$ & 8.000 \\
$(-43.3787,0.2315,897.7080)$ & 3.042 & $(-0.0051,-0.0024,600.6431)$ & \\
\hline
\end{tabular}

为了实验结果直观化，对上述数据进行处理，如下图9所示: 


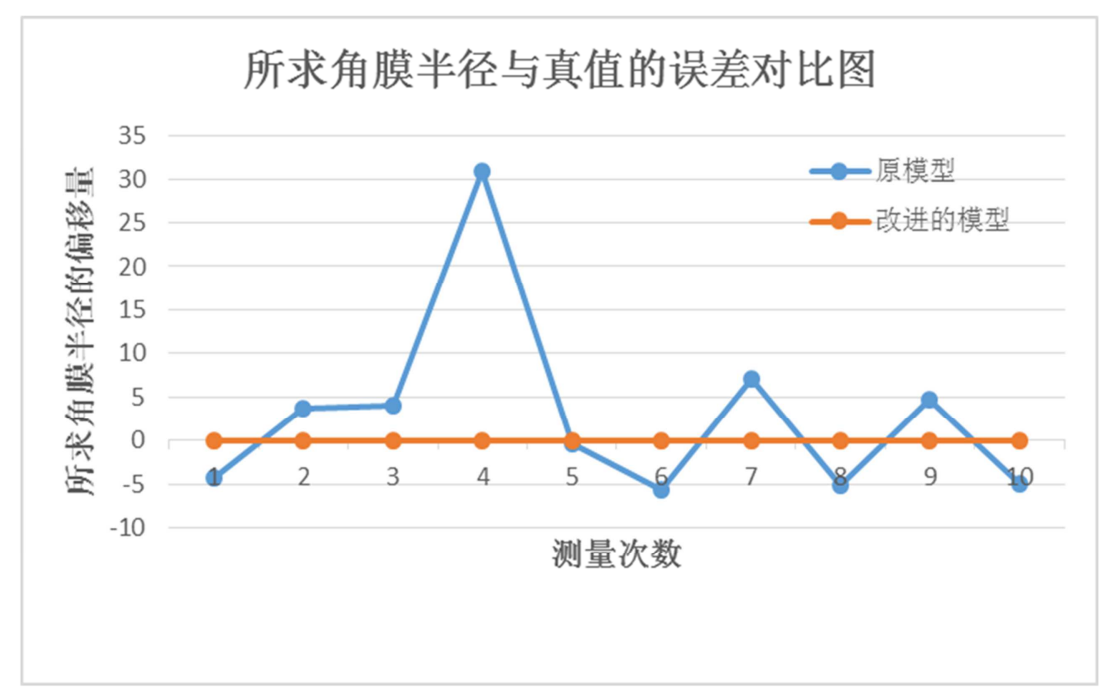

图9 角膜半径与真值误差对比图。

由表 1 , 表 2 和图 9 可知, 本文提出的方法可较精确的 估计出角膜参数。当在光源坐标中加入 $2 \mathrm{~mm}$ 的噪声, 即模 拟实物系统中光源位置标定存在 $2 \mathrm{~mm}$ 以内的误差时, 原模 型估计出的角膜参数即角膜曲率中心和角膜曲率半径存 在较大的偏差, 而改进之后的模型能够较准确的估计出角 膜参数。

由实验可知本文改进的角膜参数估计模型对一定范 围内的图像噪声具有较强的鲁棒性。

\section{5. 结论}

视线追踪技术作为最前沿的人机交互方式之一，是一 个多学科交叉, 涉及众多专业知识的研究课题, 在科学研 究, 商业, 军事等领域应用广泛。3-D视线追踪技术走向 实用级别, 必须提高其准确性和鲁棒性。本文对现有的角 膜参数估计算法进行了研究, 并针对标定精度高, 存在病 态的问题提出了一种基于最小误差法的角膜参数估计方 法, 先估计出角膜外表面半径, 在增加角膜约束下去估计 角膜曲率中心, 并通过实验验证了此方法具有较好的准确 性和鲁棒性。

\section{致谢}

本文为北京市自然科学基金（4172040）和交通运输 车辆运行安全技术重点实验室开放项目的阶段性成果之 一。感谢光电测量研究室对本文实验在图像采集过程提供 的硬件支持。

\section{参考文献}

[1] 胡大正. 自然光下基于单目摄像头的视线跟踪算法研究 [D]. 华南理工大学,2016。

[2] 梅涛.单相机视线跟踪系统研究[D]. 华中科技大学,2009。
[3] 武媛媛. 眼动跟踪技术研究[D].西安电子科技大学, 2009。

[4] 徐兴民.视线跟踪技术及其应用研究 [D]. 南京航空航天大 学,2007。

[5] 张闯,迟健男,张朝晖,等.一种新的基于瞳孔-角膜反射技术 的视线追踪方法 [J].计算机学报,2010,33(7):1272-1285。

[6] 张琼,王志良,迟健男,等.基于平面镜面向双摄像机视线追踪 系统的标定方法 [J].光学学报,2011,31(4):192-198。

[7] Lim C J, Kim D. Development of gaze tracking interface for controlling 3D contents[J]. Sensors \& Actuators A Physical, 2012, 185(5):151-159.

[8] Amir A, Zimet L, Sangiovanni-Vincentelli A, et al. An embedded system for an eye-detection sensor[J]. Computer Vision \& Image Understanding, 2005, 98(1):104-123.

[9] Cornsweet T N, Crane H D. Accurate two-dimensional eye tracker using first and fourth Purkinje images.[J]. Journal of the Optical Society of America, 1973, 63(8):921.

[10] Zhu Z, Ji Q. Eye and gaze tracking for interactive graphic display[J]. Machine Vision \& Applications, 2004, 15(3):139-148.

[11] Chen J, Ji Q. A probabilistic approach to online eye gaze tracking without explicit personal calibration[J]. IEEE Trans Image Process, 2015, 24(3):1076-1086.

[12] Yoo D H, Chung M J. A novel non-intrusive eye gaze estimation using cross-ratio under large head motion[J]. Computer Vision \& Image Understanding, 2005, 98(1):25-51.

[13] Villanueva A, Cabeza R. A Novel Gaze Estimation System With One Calibration Point[J]. IEEE Transactions on Systems Man \& Cybernetics Part B Cybernetics A Publication of the IEEE Systems Man \& Cybernetics Society, 2008, 38(4):1123-1138.

[14] 李晓娅,岑兆丰.几何光学・像差・光学设计 $[\mathrm{M}]$. 浙江大学出版 社,2014。

[15] 迟健男,张闯,王翠娟, 等.双摄像机双光源视线追踪系统标定 方法研究[J].仪器仪表学报,2011,32(4):883-891。 